Conclusion: Compared with RA without ILD, HRQL of patients with RA-ILD was significantly impaired. Disease activity, fatigue, depression and rural residence were independent predictors of HRQL in Chinese RA-ILD.

References:

[1] Zamora-Legoff JA, Krause ML, Crowson CS, Ryu JH, Matteson EL. Patterns ofinterstitial lung disease and mortality in rheumatoid arthritis. Rheumatology (Oxford, England). 2017;56(3):344-50

Disclosure of Interests: None declared

DOI: 10.1136/annrheumdis-2020-eular.6249

\section{AB1331-HPR ACTIVE DISEASE ACTIVITY IN ANKYLOSIS SPONDYLITIS: WORSE OUTCOMES AND POORER HR-QOL}

W. Zhou ${ }^{1}$, M. He ${ }^{1}$, R. Zhao ${ }^{1}$, C. Dong ${ }^{1}$, Z. Gu ${ }^{1} .{ }^{1}$ Affiliated Hospital of Nantong University, Nantong, China

Background: Ankylosing spondylitis (AS) is a chronic inflammatory disease that mainly affects the sacroiliac joints and the spine, resulting in decline in quality of life[1,2]. Poor QoL is significantly related to high disease activity[3]. However, there is no systematic report on which prognosis indicators are affected by disease activity in AS patients.

Objectives: This study aimed to evaluate the patient-reported outcome measures and health-related quality of life (HR-QoL) in AS patients defined on the basis of the Bath Spondylitis Ankylosing Disease Activity Index (BASDAI).

Methods: 204 AS patients were involved in this study. A serious of questionnaires were used to overall assess AS patients, which include: Bath Ankylosing Spondylitis Disease Activity Index (BASDAI), Bath Ankylosing Spondylitis Metrology Index (BASMI), Bath Ankylosing Spondylitis Functional Index (BASFI), the $10 \mathrm{~cm}$ Visual Analog Scale (VAS), the Self-Rating Anxiety Scale (SAS), the Self-Rating Depression Scale (SDS), the Pittsburgh Sleep Quality Index (PSQI), the Health Assessment Questionnaire-Disability Index (HAQ-DI), the Fatigue Severity Scale(FSS) and the Short Form 36 Health Survey (SF-36). Independent samples t-test, Mann-Whitney U-test, Chi-square analysis and Pearson /Spearman correlation were used to analyze the data.

Results: The results demonstrated $31.4 \%$ AS patients were in active disease activity stage. Active AS patients were older, unemployed, and had less exercise therapy than stable AS patients. Besides, AS patients with active disease activity presented more severe pain $(P<0.001)$, poor physical function $(P<0.001)$ and spinal mobility $(P<0.001)$. They were more anxious $(P<0.001)$, depressed $(P<0.001)$ and had more sleep disturbance $(P=0.001)$. Compared with active AS patients, stable AS patients had more leukocytes $(P=0.040)$, lymphocytes $(P=0.002)$, erythrocytes $(P=0.001)$ and hemoglobin $(P<0.001)$. Active disease activity had a significant impact on all dimensions of quality of life in AS patients $(P<0.001)$.

Conclusion: These findings suggested that medical personnel should pay more attention to active AS patients and make effective interventions to improve quality of life.

References:

[1] Exarchou S, Lindstrom U, Askling J, Eriksson JK, Forsblad-d'Elia H, Neovius M, Turesson C, Kristensen LE, Jacobsson LT (2015) The prevalence of clinically diagnosed ankylosing spondylitis and its clinical manifestations: a nationwide register study. Arthritis research \& therapy 17:118. doi:10.1186/ s13075-015-0627-0

[2] Qian Q, Xu X, He H, Ji H, Zhang H, Ding Y, Dai SM, Zou Y, Zhu Q, Yang C, Ye S, Jiang L, Tang JP, Tong Q, He D, Zhao D, Li Y, Ma Y, Zhou J, Yuan Z, Zhang J, Jin L, Zhou X, Reveille JD, Zou H, Wang J (2017) Clinical patterns and characteristics of ankylosing spondylitis in China. Clinical rheumatology 36 (7):1561-1568. doi:10.1007/s10067-017-3660-3

[3] Huang JC, Qian BP, Qiu Y, Wang B, Yu Y, Zhu ZZ, Hu J, Qu Z (2017) Quality of life and correlation with clinical and radiographic variables in patients with ankylosing spondylitis: a retrospective case series study. BMC musculoskeletal disorders 18 (1):352. doi:10.1186/s12891-017-1711-1

Acknowledgments: Thanks to all the authors for their efforts and thanks to all members of the Department of Rheumatology of Affiliated Hospital of Nantong University for their helpfulness in the acquisition of data.

Disclosure of Interests: None declared

DOI: 10.1136/annrheumdis-2020-eular.6288

\section{AB1332-HPR ASSESSING THE PATIENT EXPERIENCE OF LUPUS NEPHRITIS: DEVELOPMENT OF A CONCEPTUAL MODEL AND REVIEW OF EXISTING PATIENT- REPORTED OUTCOME (PRO) MEASURES}

P. Berry ${ }^{1}$, K. Burrows ${ }^{2}$, R. Hall ${ }^{2}$, A. Gater ${ }^{2}$, H. Bradley², A. Ward², C. Tolley², P. Delong ${ }^{1}$, E. C. Hsia ${ }^{3} .^{1}$ Janssen Global Services LLC, Horsham, United States of America; ${ }^{2}$ Adelphi Values Ltd, PCO, Bollington, United Kingdom; ${ }^{3} J a n s s e n$ Research \& Development LLC, Spring House, United States of America
Background: Lupus nephritis (LN) is an autoimmune disease characterized by inflammation of the kidneys as a result of systemic lupus erythematosus (SLE). Approximately $50 \%$ of SLE patients will develop LN, which is considered to be one of the most severe manifestations of SLE and the leading cause of morbidity and mortality in SLE. While there is ample existing evidence on disease experience and PROs used in extra-renal SLE, little research has been done in LN. Qualitative interviews with patients can help identify concepts that are both important and relevant to the patient. In order to effectively evaluate treatment benefit, it is critical that PRO measures used to assess such concepts and define clinical trial endpoints are fit for purpose and have strong evidence of content validity in the specific context of use.

Objectives: The objective of this study was to understand the patient experience of $L N$ and to identify and characterize the signs and symptoms of $L N$ and their impact on health-related quality of life (HRQoL) through the development of a disease-specific conceptual model. This model was then used to evaluate the content validity of existing PRO measures available for use in $\mathrm{LN}$.

Methods: A structured literature search was conducted in Medline, Embase and PsycINFO to identify qualitative research articles documenting the patient experience of LN. PRO measures developed or commonly used to assess patient experiences of LN were also identified. Semi-structured concept elicitation interviews were conducted with 15 adult patients in the US with a clinician-confirmed diagnosis of LN (defined in accordance with established clinical guidelines). Supplementary qualitative data were also collected from a review of publicly available online blogs/forums. Findings were used to inform the development of a conceptual model detailing the impact of LN signs, symptoms and HRQoL and evaluate the validity of existing measures used within $\mathrm{LN}$.

Results: Searches revealed a paucity of qualitative research conducted with LN patients, supporting the need for prospective research in LN. Consistent with existing literature in SLE, the core signs and symptoms identified from the qualitative literature review, interviews and blog/forum review included joint pain, fatigue, joint stiffness, swelling (particularly in the extremities) and skin rashes. LN patients also reported urinary frequency, urgency, foamy urine and blood in their urine. Disease impact on physical functioning, activities of daily living emotions, social life, work/finances and sleep were reported. PRO measures commonly used to evaluate patient experiences in LN included the SF-36 LupusQOL, LupusPRO, SLE Symptom Checklist, KDQoL and KSQ. Conceptual mapping of instruments against the newly developed conceptual model (Fig ure 1) highlighted that no single measure provides a comprehensive assessment of all symptoms/impact important to LN patients. Furthermore, items are largely focused on impact of symptoms with few items on symptom severity.

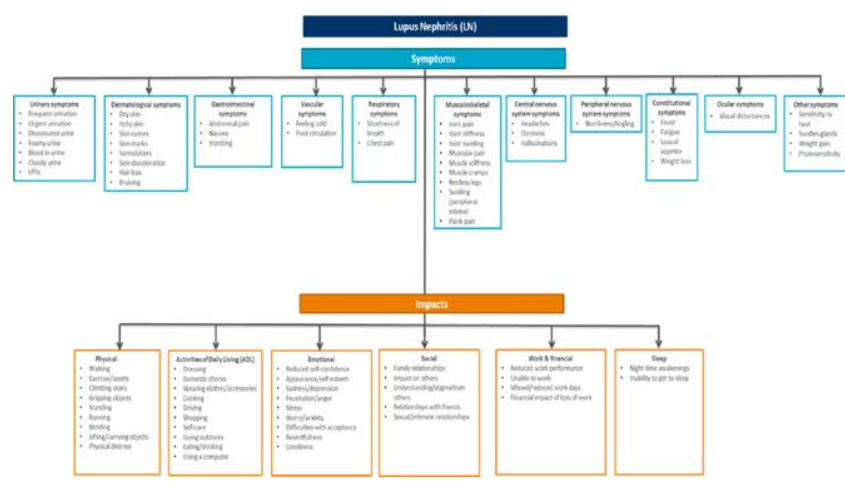

Figure 1. Conceptual model of lupus nephritis symptoms and associated impacts

Conclusion: The presentation of signs and symptoms in LN patients appears similar to those reported in extra-renal SLE populations, with the addition of swelling and urinary symptoms. Qualitative research with LN patients guided the development of a comprehensive LN conceptual model outlining the disease experience from the patients' perspective. These insights can be useful to inform PRO measurement strategies for clinical trials in LN.

Acknowledgments: With thanks to Dr. Betty Diamond and Dr. David Wofsy for their collaboration and helpful insights

Disclosure of Interests: Pamela Berry Employee of: Janssen, Kate Burrows Consultant of: Adelphi Values a health outcomes research company commissioned by Janssen to conduct the research reported in this abstract, Rebecca Hall Consultant of: Adelphi Values a health outcomes research company commissioned by Janssen to conduct the research reported in this abstract., Adam Gater Consultant of: Adelphi Values a health outcomes research company commissioned by Janssen to conduct the research reported in this abstract, Helena Bradley Consultant of: Adelphi Values a health outcomes research company commissioned by Janssen to conduct the research reported in this abstract, Amy Ward Consultant of: Adelphi Values a health outcomes research company 
commissioned by Janssen to conduct the research reported in this abstract, Chloe Tolley Consultant of: Adelphi Values a health outcomes research company commissioned by Janssen to conduct the research reported in this abstract, Patricia Delong Employee of: Janssen, Elizabeth C Hsia Shareholder of: Johnson \& Johnson, Employee of: Janssen Research \& Development, LLC DOI: 10.1136/annrheumdis-2020-eular.5634

\section{AB1333-HPR FIBROMYALGIA SYNDROME IN MEDICAL STUDENTS}

I. Akcin ${ }^{1}$, M. Gur ${ }^{2}$, R. Piskin Sagir ${ }^{2}$, B. Oz ${ }^{2}$, A. Karatas ${ }^{2}$, S. S. Koca ${ }^{2}{ }^{1}$ Firat University, Elazig, Turkey; ${ }^{2}$ Firat University, Rheumatology, Elazig, Turkey

Background: At the university, students begin to be responsible for their own life decisions and lifestyles. First year students are required to adapt especially to a new learning environment and to cope with the academic and social demands of vocational education. High academic expectations are stressful and can pose a risk to students mental and physical health. Anxiety and depression are among the most common psychiatric problems among students.

Objectives: The aim of this study is to evaluate the prevalence of fibromyalgia syndrome (FMS) in medical students and to compare students from engineering faculty.

Methods: 392 (284 faculty of medicine, 108 faculty of engineering) students selected from Firat University Faculty of Medicine and Engineering were included in the study. Hospital Anxiety and Depression Scale (HADS) forms were filled in for all participants. Anxiety and depression among students of medical and engineering were examined. Moreover, 2016 ACR FMS classification criteria was used to select the student who have FMS.

Results: In our sample, 185 (47.1\%) and 207 (52.9\%) of participants were male and female, respectively. HADS anxiety and HADS depression scores were significantly higher in engineering students than in medical students (mean HADS anxiety and depression scores were 9.07; 10.29, $\mathrm{p}=0.007$ and $7.61 ; 8.52, \mathrm{p}=$ 0.039 , respectively). While a significant difference was found among medical and engineering students in terms of HADS anxiety and depression scores in men $(p<0.001$ and $p=0.006)$, no significant difference was found in women $(p=0.676$ and $p=0.278)$. On the other hand, $46(16.1 \%)$ of medical students and $13(11.7 \%)$ of students from engineering faculty have FMS $(p=0.170)$.

Conclusion: FMS prevalences are similar in the medical students and students from engineering faculty. However, anxiety and depression are more common among male engineering students than medical students. This may be related to future employment anxiety among students from engineering faculty.

References:

[1] Cheung T, et al. Depression, Anxiety and Symptoms of Stress among Baccalaureate Nursing Students in Hong Kong: A Cross-Sectional Study. Int J Environ Res Public Health. 2016 Aug 3;13(8).

Disclosure of Interests: None declared

DOI: 10.1136/annrheumdis-2020-eular.5842

\section{AB1334-HPR BARRIERS AND FACILITATORS TO PHYSICAL ACTIVITY IN JUVENILE IDIOPATHIC ARTHRITIS (JIA): A SCOPING REVIEW}

K. O Donoghue ${ }^{1}$, L. Larkin ${ }^{2,3} .{ }^{1}$ University of Limerick, Limerick, Ireland; ${ }^{2}$ Health Research Institute, University of Limerick, Castletroy, Ireland; ${ }^{3}$ University of Limerick, School of Allied Health, Faculty of Education \& Health Sciences, Castletroy, Ireland

Background: Physical activity is an important aspect in the management of JIA (1). However physical activity levels are low in this population (2). Limited research has been conducted to identify definitive barriers and facilitators to physical activity in children and adolescents who have JIA.

Objectives: The objective of this scoping review was to identify the common barriers and facilitators to physical activity in JIA.

Methods: Original studies, either quantitative or qualitative, including participants with a diagnosis of JIA, who were under 18 years of age were included. Two independent reviewers carried out a search of the literature and full text reviews of papers to determine eligibility for inclusion. The Critical Skills Appraisal Programme (CASP), Appraisal tool for Cross-Sectional Studies (AXIS) and Downs and Black critical appraisal tools were used to assess the quality of the included research articles.

Results: Eighteen studies were included in the review. The included studies were of a variety of low, moderate and high quality. The synthesis of the data identified pain to be the most common barrier and the modification of physical activities to the need of the individual to be the most common facilitator to physical activity in JIA.

Conclusion: Identifying the most common barriers and facilitators to physical activity allows clinicians to apply better management strategies when treating an individual with JIA. Our findings demonstrate the need for further research in this area to assist increasing physical activity participation for children and adolescents who have JIA.

\section{References:}

[1] Kuntze, G., Nesbitt, C., Whittaker, J.L., Nettel-Aguirre, A., Toomey, C., Esau S., Doyle-Baker, P.K., Shank, J., Brooks, J., Benseler, S., Emery, C.A. (2018) 'Exercise Therapy in Juvenile Idiopathic Arthritis: A Systematic Review and Meta-Analysis', Archives of Physical Medicine and Rehabilitation, 99(1), 178-193

[2] Bos, G.J.F.J., Lelieveld, O.T.H.M., Armbrust, W., Sauer, P.J.J., Geertzen, J.H.B., Dijkstra, P.U. (2016) 'Physical activity in children with Juvenile Idiopathic Arthritis compared to controls', Pediatric Rheumatology, 14(1), 42.

Disclosure of Interests: None declared

DOI: 10.1136/annrheumdis-2020-eular.3550

\section{AB1335-HPR HEALTH PROFESSIONALS' PERSPECTIVE ON THE BENEFITS AND RISKS OF LOW-DOSE GLUCOCORTICOIDS IN RHEUMATOID ARTHRITIS - AN INTERNATIONAL SURVEY OF 444 HEALTH PROFESSIONALS}

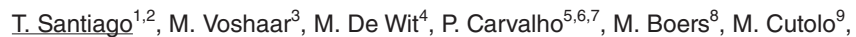
F. Buttgereit ${ }^{10}$, J. A. P. Da Silva ${ }^{1,2} \cdot{ }^{1}$ Centro Hospitalar e Universitário de Coimbra, Rheumatology, Coimbra, Portugal; ${ }^{2}$ University of Coimbra, Faculty of Medicine, Coimbra, Portugal; ${ }^{3}$ University of Twente, Department of Psychology, Health and Technology, Enschede, Netherlands and Stichting Tools Patient Empowerment, Amsterdam, Netherlands; ${ }^{4}$ Stichting Tools Patient Empowerment, Amsterdam, Netherlands; ${ }^{5}$ Centro Hospitalar Universitário do Algarve, Rheumatology, Faro, Portugal; ${ }^{6}$ Lisbon Academic Medical Centre, Lisbon, Portugal; ${ }^{7}$ Algarve Biomedical Center, Faro, Portugal; ${ }^{8}$ Department of Epidemiology and Biostatistics; and Amsterdam Rheumatology and Immunology Center, Amsterdam University Medical Centers, Vrije Universiteit, Amsterdam, Netherlands; ${ }^{9}$ Research Laboratory and Academic Division of Clinical Rheumatology, Department of Internal Medicine, University of Genova, IRCCS Polyclinic Hospital San Martino, Genova, Italy; ${ }^{10}$ Department of Rheumatology and Clinical Immunology, Charité University Medicine Berlin, Berlin, Germany

Background: The Glucocorticoid Low-dose Outcome in Rheumatoid Arthritis Study (GLORIA) is an international investigator-initiated pragmatic randomized trial designed to study the effects of low-dose glucocorticoids (GCs) in elderly patients with Rheumatoid Arthritis (RA).

The research team is also committed to promote a better understanding of the risks and benefits of these drugs among health professionals and patients. In order to achieve these goals, it is important to assess the current ideas and concerns of patients regarding GCs.

Objectives: To evaluate the current patient perspective on the efficacy and risks of GCs in RA patients who are or have been treated with GCs.

Methods: Patients with RA completed an online survey (with 5 closed questions regarding efficacy and safety) presented in their native language. RA patients were recruited through a variety of patient organizations representing three continents. Patients were invited to participate through national patient organizations. In the USA, patients were also invited to participate through MediGuard.org. Participants were asked for their level of agreement on a 5-point Likert scale.

Results: 1344 RA patients with exposure to GCs, from Brazil, USA, UK, Portu gal, Netherlands, Germany and 24 other countries** participated: $89 \%$ female, mean age (SD) 52 (14) years and mean disease duration 13 (11) years. The majority of participants (84\%) had $\geq 10$ years of education. The duration of GCs exposure was 1.6 (4.2) years. The majority of participants had read articles or pamphlets on the benefits or harms of GC therapy.

Regarding GCs efficacy (table 1), high levels of endorsement were found: about $2 / 3$ of patients considered that GCs as very useful in their case, more than half considered that GCs were effective even at low doses, and agreed that GC improved RA symptoms within days.

Regarding safety (table 1), 1/3 of the participants reported having suffered some form of serious adverse events (AEs) due to GCs, and $9 \%$ perceived this as "life-threatening. Adverse events had a serious impact on quality of life, according to about $1 / 3$ of the respondents.

Conclusion: Patients with RA exposed to GC report a strong conviction that GCs are very useful and effective for the treatment of their RA, even at low doses. This is accompanied by an important prevalence of serious AEs. Understanding the patient perspective can improve shared decision-making between patient and rheumatologist.

Funding statement: This project has received funding from the European Union's Horizon 2020 research and innovation programme under grant agreement No 634886 . 